\title{
Task-based Language Teaching (TBLT) in Asian EFL classes: Challenges and Strategies
}

\author{
Yi Ji $i^{1,2}$ \\ ${ }^{1}$ Monash University, Melbourne, Australia \\ ${ }^{2}$ Shandong Youth University of Political Science, Jinan, Shandong, China \\ sdjiyi@163.com
}

Keywords: TBLT, EFL classes, Asian context, implementation challenges and solutions

\begin{abstract}
The last two decades have witnessed an increasing and extensive application of taskbased language teaching (TBLT) in the EFL classes around the Asian countries. Despite its popularity, however, there are a variety of studies exploring and displaying the challenges and problems in its implementation in Asian classes. This paper reviews research addressing the problems in the implementation of TBLT in Asian EFL classes and their according solutions. More importantly, the paper attempts to investigate the mismatches between the pedagogic concepts underlying TBLT and the sociocultural contexts in Asia. This will help educators and language teachers in Asia to attach more importance to the contextual differences when applying TBLT to the local educational settings, both in Asia and the other regions around the world.
\end{abstract}

\section{Introduction}

The last two decades have witnessed an increasing and extensive application of task-based language teaching (TBLT) in the EFL classes around the Asian countries. There have been some official supports for this teaching approach in government curriculum reform for English teaching, illustrated in Hong Kong [1] and China [2]. In Japan, the Task-based Learning Special Interest Group of the Japanese Association for language teachers was established in 2010 [3], and the increase in its membership also reveals the more attention paid on this teaching method in this Asian country. The increasing popularity of TBLT in Asia can be attributed to the requirement for a quality-oriented education model [4] in EFL context, in which the students can develop a wide range of all-rounded abilities besides the foreign language competence. It demands a change from the teacher-centered approach and mere transition of language knowledge [5] in the traditional teaching methods specialized in Asia, such as grammar translation, presentation-practice-production and audio-lingual methods, to a communicative approach like TBLT where the learners are required to "use language, with emphasis on meaning, to attain an objective" [6].

Despite its popularity, however, there are a variety of studies exploring and displaying the challenges and problems in the implementation of TBLT in Asian countries. This dilemma can be attributed to a number of reasons, the most convincing one being the varying definition of the task in TBLT.

Defining task in TBLT has been the subject of much debate. Nunan [7], Samuda and Bygate [8], and Prabhu [9] respectively give definition of a task by emphasizing its pedagogic meaning, its essential characteristics and its cognitive process, which makes the contextual implementation of this pedagogy more complicated. Nunan [10] further defines the task in terms of its six necessary components:

The task is a piece of meaning focused work involving learners in comprehending, producing and/or interacting in the target language, and tasks are analyzed or categorized according to their goal, input data, activities, settings and (teacher and learner) roles.

To be specific, goals of a task can be in relation to linguistic, communicative, sociocultural or cultural outcome. Input of a task can involve spoken, written and visual materials exposed to learners to assist their task performance. Activities are what learners actually do with the input to 
complete the tasks. Settings refer to the classroom management for the task. Teacher roles and learner roles are the roles that the teacher and learners respectively take in the entire process of TBLT.

Considering the logical category and description of the task and TBLT in terms of the six components, in this review, the problems in the implementation of TBLT in Asian EFL classes are analyzed and categorized according to these six components. For the readers' convenience, the according solutions for each problem are analyzed just after the presentation of the specific problem. With such an organization of the review structure, the purpose of this paper is to review research addressing the problems in the implementation of TBLT in Asian EFL classes and their according solutions. More importantly, the paper attempts to investigate the mismatches between the pedagogic concepts underlying TBLT and the sociocultural contexts in Asia. This will help educators and language teachers in Asia to attach more importance to the contextual differences when applying TBLT to the local educational settings, both in Asia and the other regions around the world.

\section{Inclusion and exclusion criteria}

Although there are a large amount of publications on TBLT in local Asian languages as well as unpublished documents including theses and dissertations for master's and doctor's degrees, the review here only includes published articles in English about implementation of TBLT in Asia. All the studies reviewed here met the following criteria:

The study measured the implementation of TBLT in Asian countries at all schooling levels from primary to tertiary. This criterion excluded a large number of studies which investigated TBLT on a Western basis, such as studies which measured challenges to utilize TBLT in Denmark [11], Greece [12], New Zealand [13] and America [14]. Being a language teaching approach and being limited in Asian contexts, implementation of TBLT in EFL classes becomes our major focus in this review.

Besides, the current review focuses on the problems and their according solutions in the implementation of TBLT. The criterion excluded some studies which revealed the benefits of TBLT for Asian students, although much attention were paid to the benefits of TBLT, such as to improve students' learning attainments, oral performance and motivation [15], to enhance students' language competence [16], to equip students with a higher autonomous learning ability [17], and to promote students' thinking and inductive capacity [18].

\section{Problems in the implementation of TBLT in Asia and the solutions}

TBLT had accumulated its popularity in the previous two decades in Asia as a widespread "slogan" [19] for EFL teaching. What appeared simultaneously were varying concerns about its challenges and problems in its actual implementation in the Asian context, which could be witnessed in different Asian countries and regions, like mainland China [20][21][22][23], Japan [24][25][26], Thailand [27], Hong Kong [28][29] and Taiwan [30]. Both the teachers and students perceived some confusing dilemmas in the three stages of TBLT, namely, pre-task, task cycle and language focus [31]. The articles investigated the problems and solutions via some survey, interview and observation studies, according to which the constraints impeding the implementation of TBLT could be found associated with all the six components of the tasks.

\subsection{Problems associated with the goals in tasks}

Goals refers to the expected outcome of language learners via the implementation of TBLT in their language learning, which can be in the form of linguistic, communicative, sociocultural or cultural achievements. In its implementation in Asia, however, as illustrated in the literature, the goals of tasks are rather difficult to match the contextual reality in Asian EFL classrooms, and the mismatch can be attributed to the exam culture, the emphasis on grammar and uncertain assessment of learners' performance. 
English language teaching in most Asian countries can be characterized as dominantly teachercentred, textbook-directed and memorization-based [32]. This teaching approach has been proved effective to address the exam-oriented instruction which demands the learners to follow the teacher's instructions, to apply the exercise-stuffed tactic [33], and to merely master what is emphasized in the examinations. The English examinations in most Asian countries exclusively focus on the writing and reading competence of language learning [34], with little attention paid to speaking and listening. This inclination is opposite to the communicative teaching rationale of TBLT which emphasizes the communicative competence of language learners with their improvement in the interactive abilities [35]. The mismatch of examination culture in Asia with the goals in TBLT can profoundly confuse the pedagogical instructors about how to conduct TBLT in the specific class contexts in Asia and demotivate the learners their engagement into task performance in TBLT.

Researchers have long found the fact that examinations can be a potential constraint to the implementation of TBLT [36][37][38], and the according solutions are investigated in different contexts in Asian classrooms and in the higher-level educational system. Butler [39] proposes in his study that conceptual changes toward leaning and assessment are suggested in society as a whole. A concept that language learning is not directed to examination but to the all-rounded competence of students should be embraced in the educational system as well as in the English classes. Yan [40] specifically suggested a fundamental switch of "assessment of learning" in which assessment is perceived as the principal learning purpose to "assessment for learning" in which assessment is used to promote learning. Furthermore, researchers suggest a "situated" [41] and adaptable task-based approach in the Asian EFL classrooms, namely, TBLT and the traditional teaching approaches can be integrated together, in which tasks provided to language learners can be related to examination requirements.

Another obstacle in implementing TBLT in Asia lies in some teachers' concerns on and students' resistance of the perceived lack of grammar instruction [42]. The traditional teaching approach like grammar translation method in Asian EFL classes can be characterised by a focus on grammar teaching and learning [43], with grammar being a strong element to help students improve their mastery of the reading and writing competence of the target language. In TBLT, however, some students investigated in the study by McDonough and Chaikitmongkol [44] complain the grammatical structures in the tasks are all some old ones they have already learned before, and they perceive TBLT contributes little to their grammatical improvement, thus with little effect to their language learning. This perception of students is identical to the teachers' concerns in the study by Zheng and Borg (2013) [45], which shows a doubt on the effectiveness of TBLT, with a reduced time spent on explaining grammar, to prepare students to improve their English ability. These perceptions about TBLT considering its grammar instruction hinder its implementation in Asia by reducing the confidence of both the teachers and the students in its benefits.

TBLT can provide a way, in fact, to integrate grammar instruction with meaning-focused language use through the performance of communicative tasks [46]. One useful strategy to help students get access to grammar is in the post-task language focus stage. Grammatical forms can be emphasized in the reflection of the task performance, teacher's correction of and feedback to students' grammar usage can alleviate the danger that language learners develop language fluency at the expense of language accuracy [47]. Another solution for the lack of grammar in TBLT involves focused tasks with the use and practice of a particular grammatical structure as the targeted focus [48]. Communication and students' choice of language resources can simultaneously be emphasized in the tasks, while the grammar is used in the task performance by learners as the focus of the tasks.

The final problem in the implementation of TBLT in Asia associated with task goals lies in that teachers do not have a clear understanding about how to assess learners' performance [49], which reveals the uncertainty among some teachers about the ultimate goals of TBLT. This obstacle can be interpreted by combining the former two aspects together, namely, EFL teachers are influenced by the exam culture and emphasis on grammar in the Asian contexts, which render an ineradicable 
routine to the Asian teachers to assess language learners' performance by measuring their scores in the examinations focusing on the accurate grammatical usage of the target language. This misunderstanding of TBLT obstructs the teachers' implementation in terms of unclear orientation and definition of the contents, complexity and types of the tasks.

The countermeasure to this problem involves the formal teaching training in language methodology to EFL teachers. Teachers should have a holistic and clear understanding of how to implement TBLT, and specifically, how to assess the language learners' task performance by measuring their fluency, accuracy and complexity in the language production. Fluency can be measured by the number and percentage of meaningful words in pre-determined time, where meaningful words refer to those after "excluding self-repetitions, self-corrections and any L1 utterances" [50]. Accuracy can be measured by the percentage of error-free clauses [51]. Complexity can be measured by the mean length of the main clauses to which all subordinate clauses are attached. The goal of TBLT can be inferred from this assessment system where the communicative competence serves as the fundamental units of the teaching approach while grammatical focus still arises the emphasis, which complies the overarching rationale for TBLT that learners' attention is drawn to form in the context of meaning [52].

Based on the review above, Figure 1 illustrates the problems in the implementation of TBLT in Asian EFL classes and the according solutions which are associated with goals in tasks.

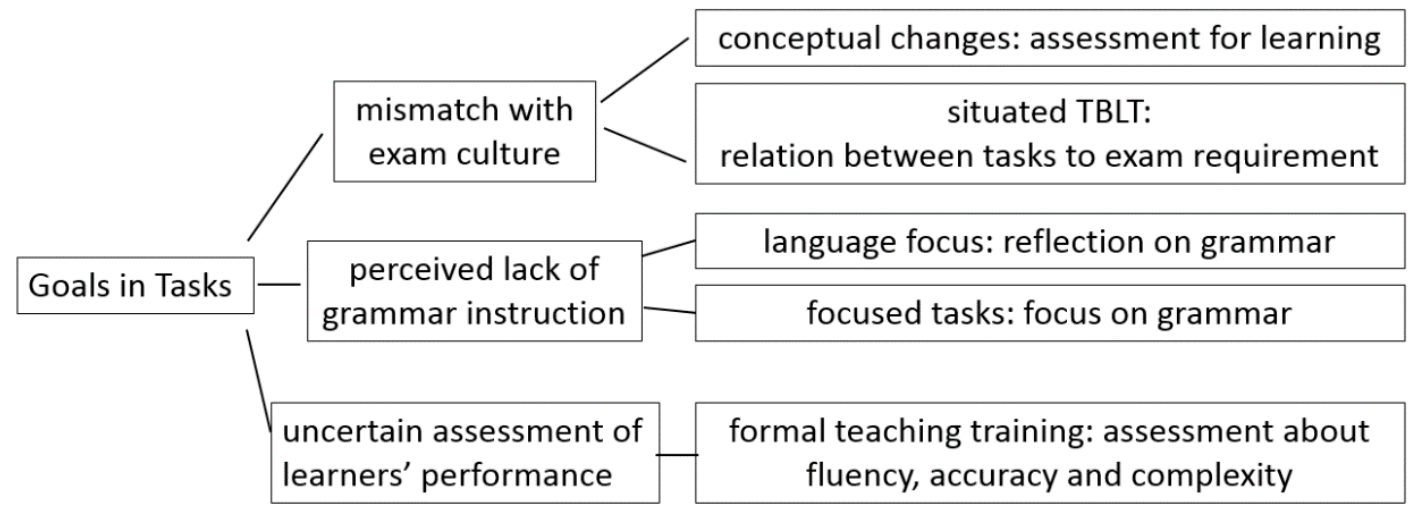

Figure 1. Problems and solutions associated with goals in tasks

\subsection{Problems associated with the input in tasks}

Input in TBLT is the materials exposed to learners before and during their task performance to assist their successful task completion, which can be in the form of spoken, written and visual materials. In its implementation in Asia, however, as illustrated in the previous studies, the preparation of task input is challenging to a majority of teachers in Asian contexts, and the obstacles can be attributed to the lack of meaningful and authentic input, and the mismatch between the task input and the textbook.

The first problem about the lack of meaningful and authentic input stems from the lack of nativelike contexts in Asian countries for English learning [53]. The meaning of authentic input refers to that materials and activities in the task can reflect the actual language use and life reality in the English-speaking countries [54]. Based on the linguistic and living environment in Asian countries, the English teachers in Asia have resources and assistance far from being sufficient for them to prepare the task input which can address the requirement of being authentic. The imported [55] or fabricate task input cannot be related to either the actual language reality in the English-speaking countries or to the language learners' daily lives in the Asian countries, the input is therefore not meaningful for the language learners to guarantee them a fruitful linguistic achievement in TBLT.

The authenticity and meaningfulness of task input can be guaranteed via teachers' effort to get access to the authentic materials both in the academic and daily life circumstance. To find audio and video materials from the internet produced by native English speakers can be of enormous benefits to ensure the authenticity of task input. In terms of the meaningfulness, Burrows [56] 
explores that the "comprehensible" input is not enough to motivate learners' involvement, rather tasks should be meaningful enough to "tap into" learners' learning styles. Besides, more opportunities to use English in real-life settings can be created to language learners [57]. Language learners in Asian countries can use computer-mediated technology to interact with people in English-speaking regions around the world, and to provide mutual peer supports in English practices like in English conversation activities.

Another problem associated with the task input lies in teacher's and students' concern about the mismatch between the task and the textbook content. Although only the research by McDonough and Chaikitmongkol [58] illustrated this concern, its side effect on the implementation of TBLT in Asia can be evident. The teacher's responsibility to complete the teaching schedule related to the textbooks can be hindered by the unrelated task input, which would in turn discourage teacher to implement TBLT in their EFL classes.

The solutions provided in this article [59][60] converge in the creation of teachers' own task input materials and selection of textbooks. Teachers can create their own task input according to the tasks, under the guidance of the targeted achievement of the tasks. Furthermore, teachers can be endowed with freedom to select the commercial textbooks. The principle should be that the textbook selection and usage is dictated by the curriculum rather than that the content of the course is dictated by the textbook. The related suggestion also can be found in the study by Laurence [61], who proposed that teachers can develop their own curriculum to use in the classroom according to their teaching experience and teaching goals. Another more feasible solution can be the more cooperation between the language teachers and other subject teachers [62]. Working together with other subject teachers, language teachers can combine their academic knowledge and language teaching skills in their preparation for the task input.

Based on the review above, Figure 2 illustrates the problems in the implementation of TBLT in Asian EFL classes and the according solutions which are associated with input in tasks.

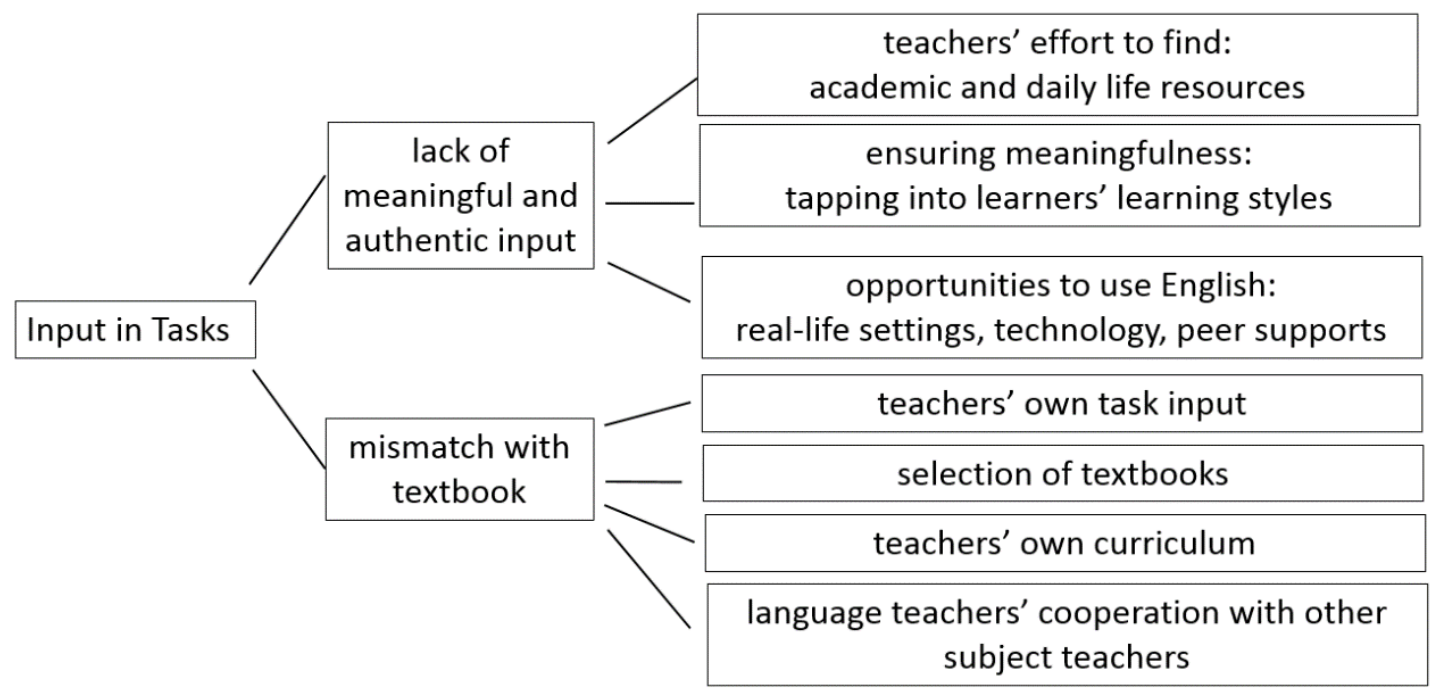

Figure 2. Problems and solutions associated with input in tasks

\subsection{Problems associated with the activities in tasks}

Activities refer to what learners actually do in the task performance, including learners' application of the target language and their engagement into the task performance. According to the literature, the problems in the implementation of TBLT in Asia associated with the activities include learners' frequent L1 use, the implementation of TBLT divergent to its rationales and the persistence of traditional teaching methods.

Willis [63] offers practical guidance for teachers' real implementation of TBLT, among which there is a requirement for the target language use. In its implementation in Asian contexts, however, a number of research [64][65] revealed the frequent but unexpected L1 use in language learners' task performance. According to Carless [66], L1 may serve social and cognitive functions in 
learners' task performance. Language learners construct mutual assistance and create collaborative dialogue for language acquisition by applying their mother tongue. This use of L1, however, can undermine the rationale of TBLT as stretching language learners' interlanguage by motivating them to engage in a communicative task. Another more evident adverse effect of L1 use is the teachers' sense of frustration and doubt about the effectiveness of TBLT.

Language teachers should have a feasible understanding [67] about learners' L1 use. It should be perceived as a learner-centered strategy with potential support to learners' language learning. In terms its risk to discourage learners to master the target language by engaging in the communicative language use, teachers should give priority to learners' needs and interests [68] and their actual language proficiency [69] to motivate leaners to engage more in the target language use in the task performance. Once they gain the sense of achievement and personal accountability [70] of applying the target language, frequent L1 use can gradually disappear in the EFL classes.

In the activities of TBLT, some problems are revealed in terms of the mismatch between its actual implementation and its rationales. In the research by $\mathrm{Hu}$ [71], some EFL teachers just passively accept TBLT as the teaching method in their classes by doing what the textbook or the teacher's manual tells them to do. They had a misunderstanding of TBLT as teaching with activities, in which they erroneously instruct students to participate in different kinds of activities with Chinese and English as the instructional language. These activities, however, without the communicative quality, are not under the rationale of TBLT.

The persistence of traditional teaching methods is also observed by some researchers in the Asian EFL classes where TBLT are implemented. Yan [72] proposed that although TBLT is implemented in the EFL classes by some teachers, the traditional teaching method remains prevalent, where TBLT appears to be a supplementary method to the gramma-translation method, and the instruction remains teacher-centered. Similarly, Cui [73] also finds that some teachers apply tasks to reinforce certain grammar knowledge or sentence structures. Although TBLT is implemented, the teaching goals remain the traditional ones. Both the teachers and students pay more attention to language forms rather than language meanings, which adheres to the rationale of traditional teaching.

The two problems associated with activities in TBLT, namely, the mismatch between language teachers' actual implementation in Asia and the principles of TBLT, and the persistence of traditional teaching methods can be both reduced by the professional development of teachers and their self-reflection and peer observation [74]. Asian teachers can get the professional training about the western-based teaching methodology to have a clear understanding about how to implement it in their EFL classes, and by working together with other colleagues by reflection and observation, they can notice some details in their implementation of TBLT necessary to be modified.

Based on the review above, Figure 3 illustrates the problems in the implementation of TBLT in Asian EFL classes and the according solutions which are associated with activities in tasks.

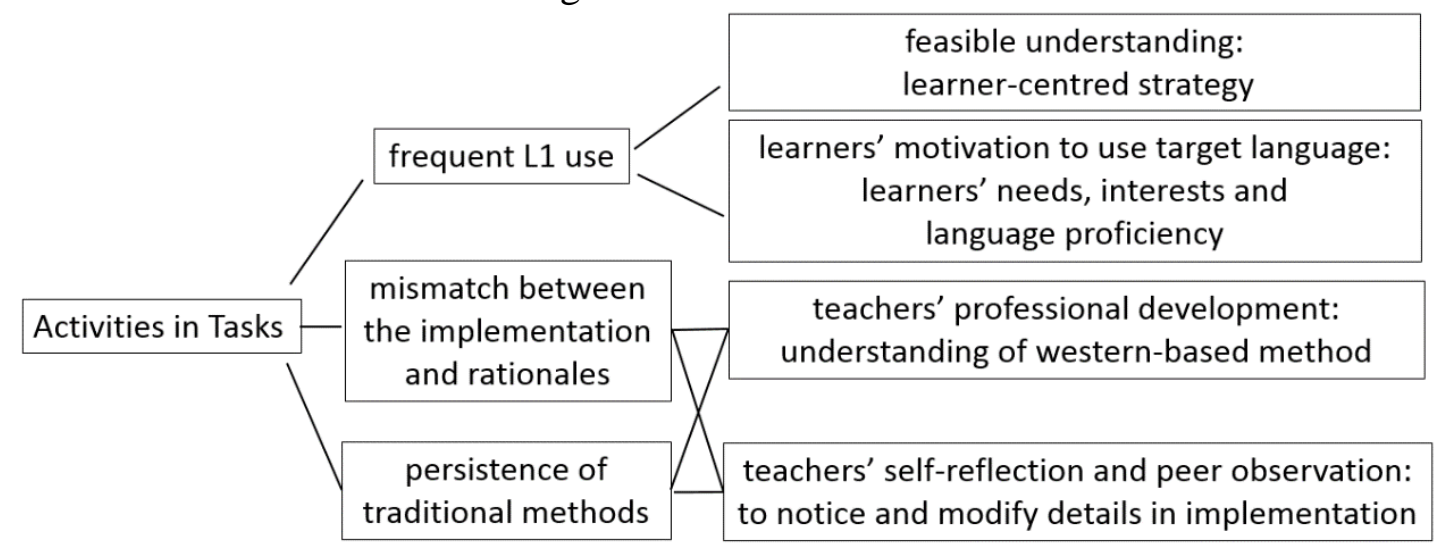

Figure 3. Problems and solutions associated with activities in tasks 


\subsection{Problems associated with the settings in tasks}

Settings in TBLT can be analyzed in terms of the classroom management for the task, about teacher's approaches to administrate the entire discipline inside the classroom, and to instruct the task performance activities during the task cycle. The problems associated with settings in the implementation of TBLT lie in large class size and limited instruction time.

The large class size appears to be a widespread problems for Asian EFL teachers of all the schooling levels. The class size ranges from 40 students to 100 in Asian classes, which challenges EFL teachers who applies TBLT with a relatively noisy classroom situation [75]. Students tend to have off-task discussions or even quarrels in the classes, leaving teachers the difficulty to keep the class disciplined [76]. By adhering to the communicative task in TBLT, teachers can be confronted with the class management problems.

Considering the demographic reality in Asian countries, it cannot be easily changed to rearrange the class size, the rational solution for this problem may involve the teachers' instruction on students in the task performance. Task types, task sequences and task complexity must be taken into account in the task design [77]. Based on the specific condition of the students, teachers can make varying choices of the tasks to enhance the classroom management. Besides, students should be trained and taught before the task performance that the principles of interaction, interdependence and individualism [78] in the TBLT process. They can be equipped with the concept that communicative discussion can be more effective when they engage more in the task-based topics and in the disciplined order.

The limited instruction time is another problem associated with settings in Asian EFL classes. In the primary, secondary and tertiary education levels, EFL lessons accounts for a small percentage of the curriculum in the school education [79]. In addition, obliged to complete the teaching schedules, teachers have even limited time to instruct students perform the tasks which are useful to improve their linguistic competence.

The solutions shown in the articles for the limited time can be initially analyzed in terms of the decrease of tasks and the increase of the time for EFL lessons. Watson [80] investigates a "mixed methodology" in which the number of tasks introduced in class are reduced, and Yang [81] also proposed that an increased amount of time devoted to English course is a vital issue. In addition, more appropriate task design can afford a smoothing process and more saved time for the implementation of TBLT in the EFL classes, which can prevent students from feeling that it is a waste of time for some boring and unnecessary tasks [82]. Only the tasks complying with the language learners' language proficiency, actual life needs and interest are the best situated ones for teachers to provide in the EFL classes.

Based on the review above, Figure 4 illustrates the problems in the implementation of TBLT in Asian EFL classes and the according solutions which are associated with settings in tasks.

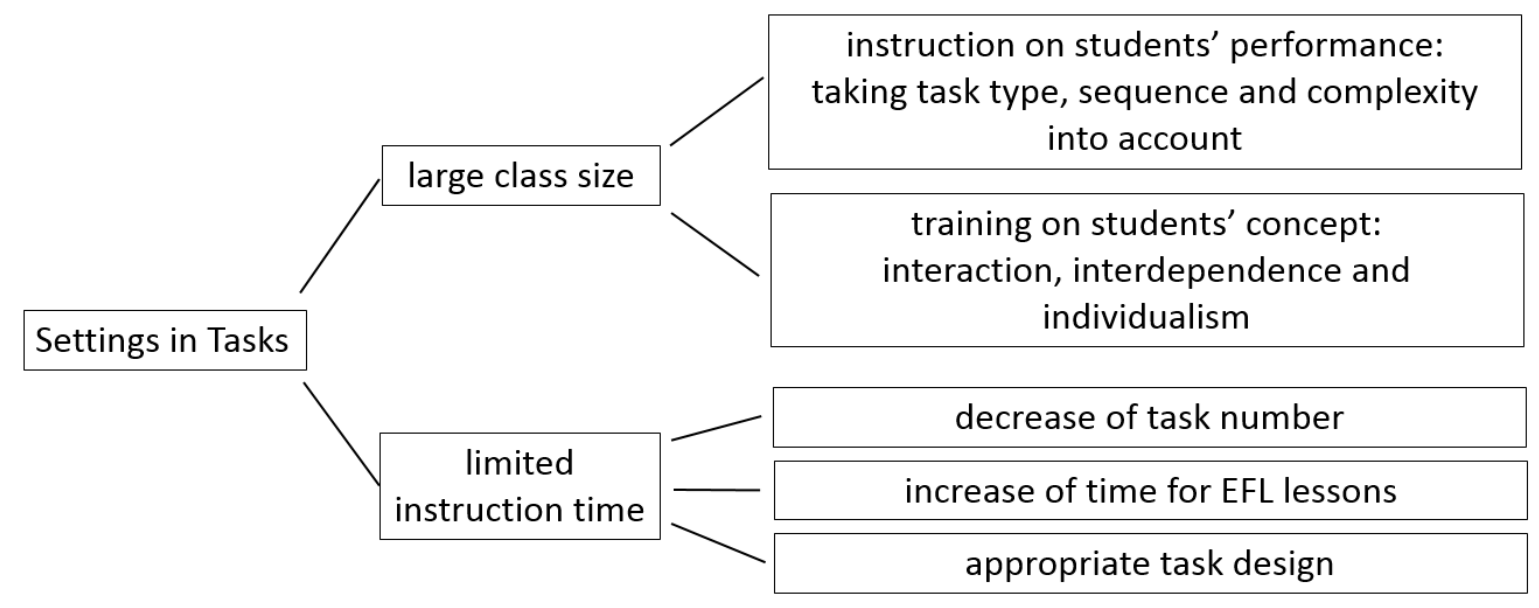

Figure 4. Problems and solutions associated with settings in tasks 


\subsection{Problems associated with the teacher roles in tasks}

Teacher roles can be analyzed in different ways in the three stages in TBLT. In the stage of pretask activities, teachers organize the tasks, prepare the linguistic input, and explain the task requirement to the learners. In the task cycle stage, teachers can be task facilitators to assist the task performance of learners. In the final post-task language focus stage, teachers give feedback to leaners about their task performance and instruct learners to focus on language applied and learnt in the task cycle stage. In the literature about the actual implementation of TBLT in Asia, however, problems are obvious in terms of the teachers' misunderstanding of TBLT, the lack of their proficiency and their involvement.

With the thriving of TBLT in Asian countries, increasing Asian teachers apply this communicative teaching method in their EFL classes. According to the literature, however, their perception of TBLT is to certain extent erroneous. Some teachers equate TBLT with learners' oral pair and group work [83], with their major attention paid on communicative activities among the language learners. These activities in which students are just instructed to speak English without attention paid to their language use, are not related to students' actual linguistic competence improvement. This misunderstanding diverges from the rationale of TBLT that pedagogical primacy is given to meaning [84] and language learners' communicative competence. In some other teachers' conception of TBLT, they hold a negative denial [85] on TBTL by doubting that students cannot really master the language by "playing games", from which we can conclude their confusion between pedagogical tasks and self-designed games.

This problem can be settled by the professional training of teachers and a hybrid teaching method supporting them. Burrows [86] suggested further professional development for teachers on how to carry out the elements of TBLT. A situated task-based approach for TBLT [87] can also draw on the cultures and settings in Asian EFL classes to address teachers' misconception of it, in which tasks can be related to the context-specific traditional teaching method and in which grammar instruction can also be emphasized in both the pre-task and post-task stages.

Some Asian teachers are limited to apply TBLT in their EFL classes because of their own quality and personal characteristics [88]. The relatively unpredictable class management condition demands more linguistic mastery and pedagogical wisdom than the traditional teaching methods where teaching is structured and planned. Some Asian teachers who are used to the traditional teaching methods, therefore, do not feel confident and comfortable to take risks to examine the effectiveness of TBLT in their own EFL classes.

Training on teachers' classroom talk and pedagogical instruction can be efficient to solve these problems. Training on teachers' classroom talk [89] can include systematic guidance on their prosodic features (stress, intonation and pronunciation), their adjusted speed of speeches, their repeating on the critical messages and their gestures and facial expressions. With a priority given to their classroom talk, teachers can pay more attention to their use of targeted language, their interaction with students and their smoothing conduction of tasks. Asian teachers can also be encouraged by some professional training focusing on the pedagogical methods and teaching philosophies about TBLT [90], such as how to arrange the task sequence, how to select proper task types and how to design the task complexity according to the targeted linguistic competence and learners' proficiency. By this training, teachers can realize that even with limited abilities, they could also implement TBLT in a successful way through comprehensive planning and appropriate design.

Compared with the traditional roles like the guide in English learning strategies and the organizer of the class activities, fewer teachers take the role as participants into the communicative tasks in TBLT [91]. Their students, however, complain the lack of teachers' assistance and feedback, which hinders their subsequent performance in TBLT.

Teachers' attention should be appropriately given to every step in TBLT [92], especially in the task cycle stage, teachers are supposed to actively participate to facilitate learners' task performance. Based on the study conducted by Chen and Zhang [93], to distinguish task-based interaction 
pedagogy and task-supported instruction approach can render teachers a clear perception of their different roles in TBLT. This accords with the category of its strong version [94] and weak version [95], the former regards tasks as the central component in syllabus design, while the latter demands tasks as communicative practice assisting the grammar-based syllabus. Teachers thus play different roles in these two versions of TBLT, they are required to actively participate in students' interactions to complete tasks in a collaborative method in the task-based pedagogy, and to formally instruct students to practice in the communicative tasks in the task-supported approach.

Based on the review above, Figure 5 illustrates the problems in the implementation of TBLT in Asian EFL classes and the according solutions which are associated with teacher roles in tasks.

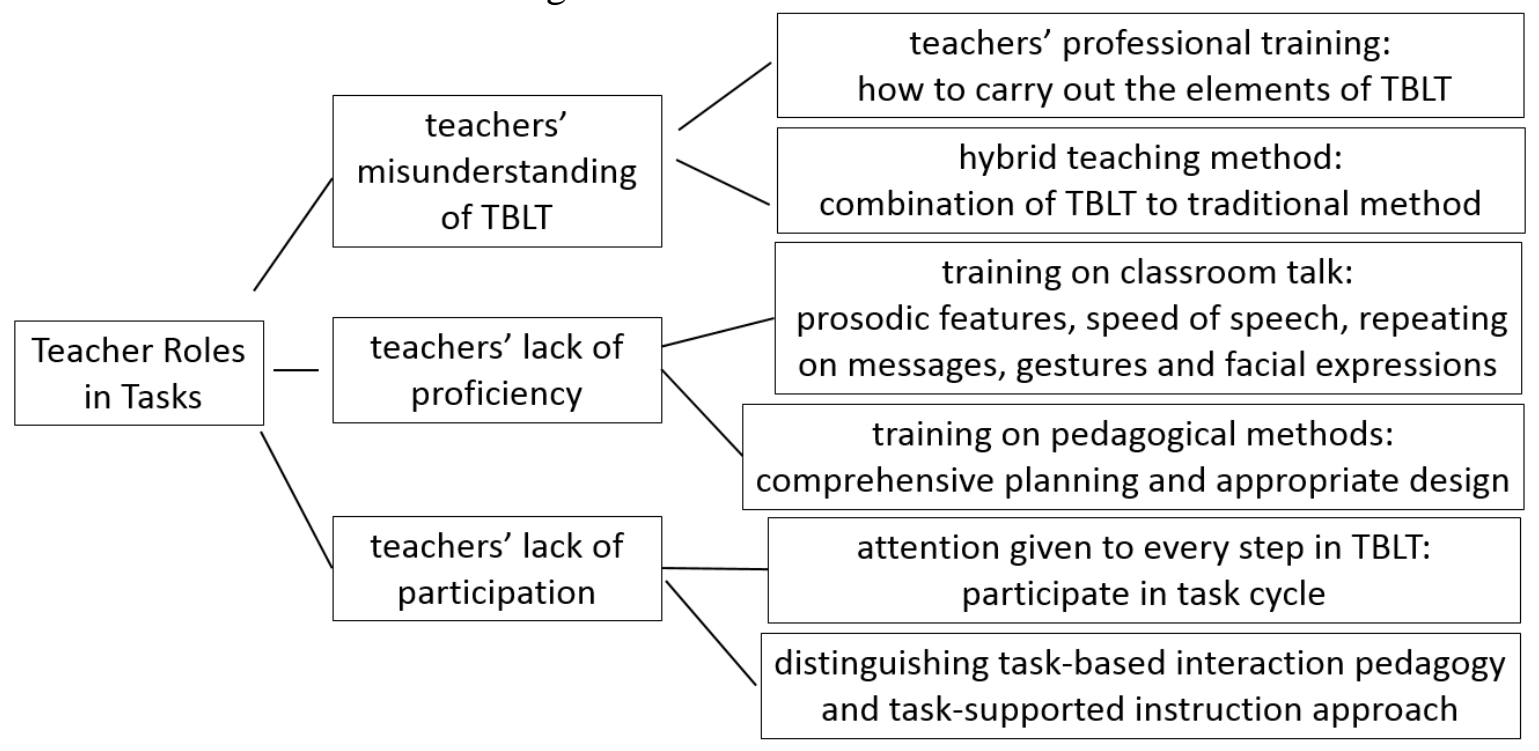

Figure 5. Problems and solutions associated with teacher roles in tasks

\subsection{Problems associated with the learner roles in tasks}

Learner roles can similarly be interpreted in terms of the three stages in TBLT, namely, to absorb the input in the pre-task stage, to perform the task by applying the target language in the task cycle stage and to reflect the task goals in the language focus stage. Problems in the implementation of TBLT in Asia associated with learner roles can also be analyzed like those with the teachers, in terms of their misunderstanding of TBLT, the lack of their proficiency and their involvement.

In the teacher-centered, exam-oriented culture in Asian countries, the student-centred and quality-oriented rationale for TBLT faces the misunderstanding from the students. Most students show their preference to and hope of following their teachers' instruction in their task performance [96], with an inclination to teacher-centred instruction evident in their misunderstanding of TBLT. In terms of the benefits of TBLT, most Asian students can merely perceive its academic contributions in enhancing their language competence and learning strategies in their exam preparation, but ignore its advantages outside classes on a daily basis [97] and complain TBLT as a waste of time blocking their exam preparation.

Students should be provided with sufficient linguistic input and structured instruction prior to their task performance. Some pre-established linguistic structures [98] included in the pre-task input can be repeatedly exercised in students' task performance, which can cater to their demands on some tangible improvement in their academic performance. In addition, students must be instructed to recognize the rationales underlying the selection of each task, and to raise the awareness that TBLT can benefit them both in their linguistic competence and their daily abilities such as collaborative work and analyzing strategies.

Another constraint for students to take their appropriate roles in TBLT is displayed in the literature as their limited abilities. The lower language proficiency is the most striking constraint for students. Students in Huang's [99] study attribute their failure in task performance to that they cannot find appropriate words in target language to express their thoughts, and that they 
consistently feel awkward to speak English together with or in front of their peer learners. TBTL, by its communicative nature, is even difficult for Asian students with lower level of communicative abilities [100] in English to participate in the task performance. The lack of their other related abilities also impede their task performance. Lack of experience in group discussion and public presentation of Asian students is one factor influencing students' passion for TBLT.

Task design and teaching methodologies in TBLT can be taken into consideration by Asian teachers to cater to the specific needs and abilities of students. To be specific, initially, tightly structured tasks such as focused tasks where students are intended to practice particular linguistic features are more suitable to language learners with lower proficiency [101], they can feel at ease when they are practicing some familiar linguistic points to gain a sense of achievement. Some short and simple tasks and hybrid tasks [102] blending newly built and previously established linguistic structures together can also be applied to alleviate the task difficulty. Besides, students can be entitled with responsibility in groups to supervise the discussion, to remind each other of the target language use and to encourage each other by peer reflection. Another impetus for students can be established by a positive atmosphere where students feel comfortable to speak freely and make mistakes.

Some students complain that their peer performers' poor pronunciation and uninteresting contents demotivate their participation into the task performance [103]. The interaction among students thus are always observed dominated by just one or two students [104], with the majority of students just sitting there, observing others and idling their time. Even the students devoting themselves in the task performance do not actually attempt to exploit their full linguistic resources but produce language at only the minimum level of complexity to complete the demanded tasks.

Teachers can remind the students of the importance of participation in the tasks by explaining process is of more meaning than the result [105], students, on the other side, should develop their consciousness about the self-learning processes [106]. Recorders can also be used to raise students' awareness of participation in the task performance. Furthermore, students' enthusiastic engagement into the tasks can be enhanced by providing them stimulating pre-task instruction, sufficient planning time and timely task evaluation and by motivating them to work with their peers with the higher or similar language proficiency levels as themselves [107].

Based on the review above, Figure 6 illustrates the problems in the implementation of TBLT in Asian EFL classes and the according solutions which are associated with learner roles in tasks.

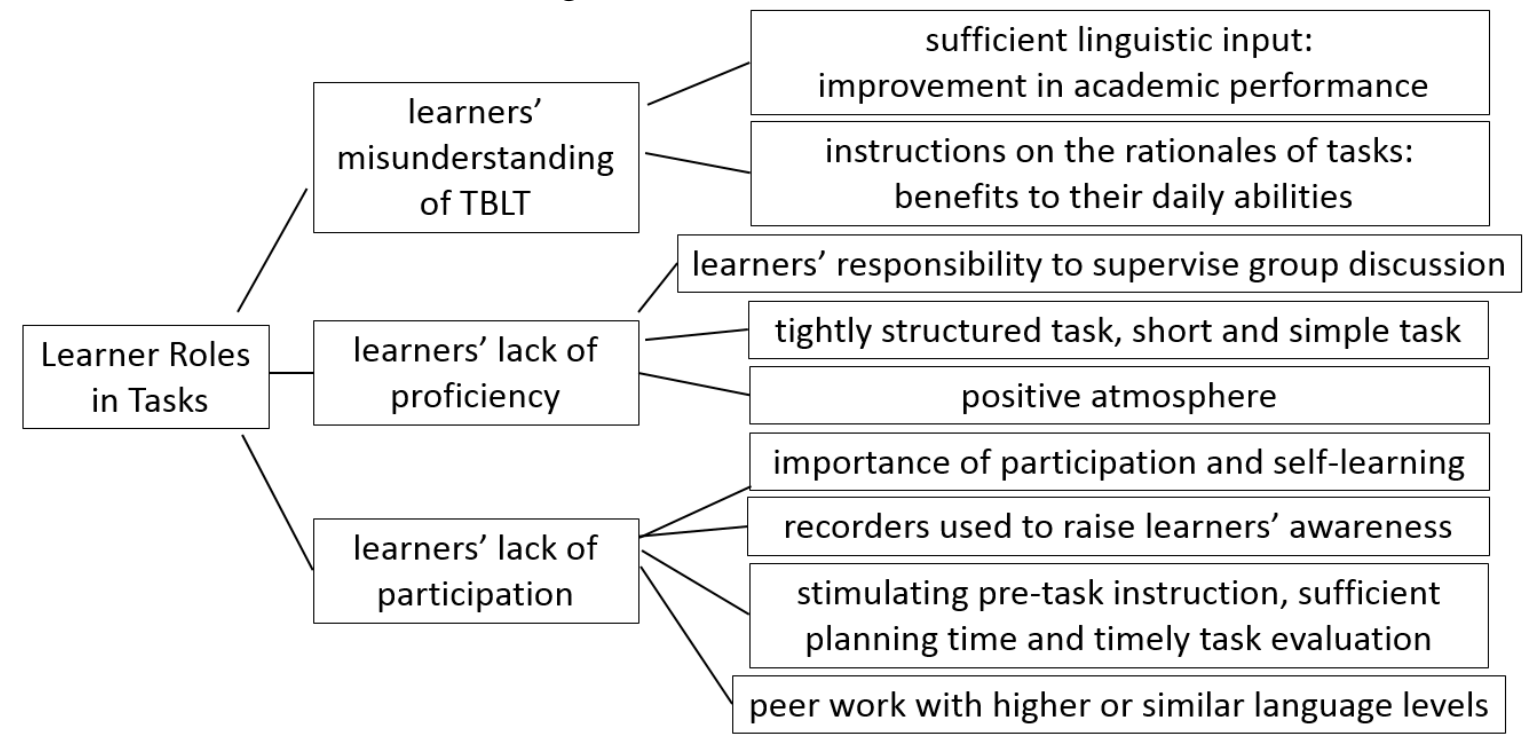

Figure 6. Problems and solutions associated with learner roles in tasks

\section{Conclusion}

Based on the literature reviewed in this study, we can tentatively conclude that TBTL has some 
problems around the socio-cultural and other contextual factors [108] in Asian EFL classes. TBTL, with its emphasis on students' interaction in the communicative activities, students' all-rounded improvement in qualities and meaningful communication in real-world context, has conflicts with Confucian heritage teacher-centred, exam-oriented and grammar-focused cultures of education in Asia. All these mismatches can be categorized in terms of the six components of tasks, which provide us with an overview about how to avoid and solute all these conflicts from the macro, meso and micro levels [109]. All the three levels in Asian school education system can contribute to the modification of TBLT into the Asian context. The curriculum developers, textbook designers and educational management authorities should revise the educational orientation to address the increasing requirement on Asian students' communicative competence in the globalization trend. The school administrators and teacher educators should afford more supports via professional training and material maintenance to assist the innovation and adaptation of TBLT. Asian teachers and students as the immediate implementers of TBLT should always bear in mind the rationales and philosophies of this teaching methodology by performing their appropriate roles in every stage of TBTL.

Our aim of revealing these problems in the implementation of TBLT in Asia is to help Asian EFL teachers to have a better understanding about the cultural adaptability of TBLT in the Asian contexts by recognizing the contextual factors which hinder the appropriate implementation of TBLT in Asia. By exploring the according solutions for these problems, this review can further help Asian teachers avoid the predicted mismatches and do some necessary modifications. These countermeasures can also be realized outside the Asian contexts to provide a sociocultural perspective to language teachers who are interested in TBLT in other regions of the world. However, taken together all what has been found in this study, these findings seem still limited and narrow. There need to be more research reporting the examination on the effectiveness of the solutions proposed in the articles reviewed here. More importantly, there need to be some research investigating the underpinning reasons for these problems from the related theories as the theoretical framework, like sociocultural theory, interaction hypothesis, input hypothesis, output hypothesis, etc. Once such research is conducted, it would provide Asian teachers with valuable instructions to implement TBLT in the specific Asian contexts.

\section{References}

[1] [38][104] Littlewood, W. Communicative and task-based language teaching in East Asian classrooms [J]. Language Teaching, 200740 243-249.

[2][24][56][69][70][77][78][86][90] Burrows, C. An evaluation of task-based learning (TBL) in the Japanese classroom [J]. English Today, 2008 24(4) 11-16.

[3][25][100][102][108] Harris, J. Teachers' Beliefs about Task-Based Language Teaching in Japan [J]. The Journal of Asia TEFL, 201613 (2) 102-116.

[4][33][34][40][65][72] Yan, C. 'We can’t change much unless the exams change': Teachers' dilemmas in the curriculum reform in China [J]. Improving Schools. 2015 18(1) 5-19.

[5][22][71][85] Hu, R. Task - Based Language Teaching: Responses from Chinese Teachers of English [J]. The Electronic Journal for English as a Second Language, 2013 16(4) 16-21.

[6] Bygate, M., Skehan, P. and Swain, M. (Eds.). Researching pedagogic tasks, second language learning, teaching, and testing. Harlow, UK: Longman, 200111.

[7] Nunan, D. Task-based language teaching. Cambridge: Cambridge University Press. 2004.

[8] Samuda, V. and Bygate, M. Tasks in Second Language learning. New York: Palgrave Macmillan. 2008. 
[9] Prabhu, N. S. Second language pedagogy. Oxford, UK: Oxford University Press. 1987.

[10] Nunan, D. Design tasks for the communicative classroom. Oxford: Oxford University Press. 198911.

[11] Bao, R. and Du, X. Implementation of task-based language teaching in Chinese as a foreign language: benefits and challenges [J]. Language, Culture and Curriculum, 2015 28(3) 291-310.

[12] Doukas, E. K. Teacher identified factors affecting the implementation of an EFL innovation in Greek public secondary schools [J]. Language, Culture and Curriculum, 1995 8(1) 53-68.

[13] East, M. Addressing the intercultural via task-based language teaching: possibility or problem [J]? Language and Intercultural Communication, 2012 12(1) 56-73.

[14] Lai, C., Zhao, Y. and Wang, J. Task-Based Language Teaching in Online Ab Initio Foreign Language Classrooms [J]. The Modern Language Journal, 2011 95(Supplementary Issue) 81103.

[15] Zhang, X. and Hung, S. A case study of exploring viability of task-based instruction on college English teaching in big-sized class [J]. Journal of Language Teaching and Research, 2013 4(4) 693-697.

[16] Youjin, K. Using pre-task modelling to encourage collaborative learning opportunities [J]. Language Teaching Research, 2011 15(2) 183-199.

[17][21][64][76][99][105] Huang, D. 2016. A study on the application of task-based language teaching method in a comprehensive English class in China [J]. Journal of Language Teaching and Research, 2016 7(1) 118-127.

[18] Zhang, L. and Li, D. Application of task-based language teachihng in college English teaching. Paper presented at International Conference on Management, Education and Social Science, 2014 126-128.

[19][36][39][54] Butler, Y.G. The Implementation of Communicative and Task-Based Language Teaching in the Asia-Pacific Region [J]. Annual Review of Applied Linguistics, 201131 36-57.

[20][49][73][88] Cui, J. EFL Teachers' Cognition and Usage of TBLT in Beijing. Master thesis. University of Alberta. 2001.

[22][59][61][107] Laurence, C. Task-based learning effects on motivation and participation in English as a second language Chinese classrooms. Master's thesis. Eastern Oregon University. 2015.

[23][82][91][92][96][103] Meng, Y. and Cheng, B. College studetns’ perceptions on the Issues of Task-based Language Teaching in Mainland China [J]. Journal of Language Teaching and Research, 2010 1(4) 434-442.

[24][53][79] Yuan, Y. Application of Task-based learning in Chinese context [J]. Theory and Practice in Language Studies, 2016 6(2) 392-397.

[26][89] Moser, J., Harris, J. and Carle, J. Improving teacher talk through a task-based approach [J]. ELT Journal, 66(1) 2012 81-88.

[27][42][44][58][59][97][98] McDonough, K. and Chaikitmongkol, W. Teachers' and Learners' Reactions to a Task-Based EFL Course in Thailand [J]. TESOL Quarterly, 2007 41(1) 107-132.

[28] Carless, D. Issues in teachers' re-interpretation of a task-based innovation in primary schools [J]. TESOL Quarterly, 200438 639-662. 
[29][37][41][46][65][66][67][87][101] Carless, D. The suitability of task-based approaches for secondary schools: Perspectives from Hong Kong [J]. System, 200735 595-608.

[30][57][62][68][74][81][106][109] Yang, J. Y. Integrating the task-based approach and the Grammar Translation Method with computer -assisted instruction on Taiwanese EFL college students' speaking performance. Doctor’s Dissertation. Alliant International University. 2008.

[31][63][94] Willis, J. A framework for task-based learning. London, UK: Longman. 1996.

[32] [43] [45] [75] [83] Zheng, X. and Borg, S. Task-based learning and teaching in China: Secondary school teachers' beliefs and practices [J]. Language Teaching Research, 2013 18(2) 205-221.

[35] Littlewood, W. The task-based approach: Some questions and suggestions [J]. ELT Journal, 200458 319-326.

[47] Skehan, P. A Framework for the Implementation of Task-based Instruction [J]. Applied Linguistics, 1996 17(1) 38-62.

[48][84][95] Ellis, R. Task-based language learning and teaching. Oxford, UK: Oxford University Press. 2003.

[50] Mochizuki, N. and Ortega, L. Balancing Communication and Grammar in Beginning-Level Foreign Language Classrooms: A Study of Guided Planning and Relativization [J]. Language Teaching Research, 2008 12(1) 11-37.

[51] Yuan, F. and Ellis, R. The effects of pre-task planning and online planning on fluency, complexity and accuracy in L2 oral production [J]. Applied Linguistics, 2003 24(1) 1-27.

[52] Long, M. Focus on form in task-based language teaching. In R. Lambert and E. Shohamy (Eds.), Language policy and pedagogy: Essays in honor of A. Ronald Walton. Philadelphia: John Benjamins. 2000 178-192.

[55] Kachru, B. B. Teaching world Englishes. In B. B. Kachru (Ed.), The other tongue: English across cultures. Urbana: University of Illinois Press. 1992 355-365.

[80] Watson, T, R. Continuing change after the innovation [J]. System, 2006 34 1-14.

[93] Chen, B. and Zhang, Y. An Experimental Study on Task-Based Interaction in Improving College Students' English Speaking Ability [J]. International Journal of Information and Education Technology, 2015 5(8) 609-614. 